This document is confidential and is proprietary to the American Chemical Society and its authors. Do not copy or disclose without written permission. If you have received this item in error, notify the sender and delete all copies.

\title{
Charge Delocalization in an Organic Mixed Valent Bithiophene is Greater than in a Structurally Analogous Biselenophene
}

\begin{tabular}{|r|l|}
\hline Journal: & The Journal of Physical Chemistry \\
\hline Manuscript ID: & jp-2014-082164.R1 \\
\hline Manuscript Type: & Article \\
\hline Date Submitted by the Author: & $24-$ Oct-2014 \\
\hline Complete List of Authors: & $\begin{array}{l}\text { Jahne, Ann; University of Basel, Department of Chemistry } \\
\text { Proppe, Jonny; University of Hamburg, Chemistry (Institute for Technical } \\
\text { and Macromolecular Chemistry) } \\
\text { Spulber, Mariana; University of Basel, Chemistry Department } \\
\text { Palivan, Cornelia; University of Basel, Chemistry Department } \\
\text { Herrmann, Carmen; University of Hamburg, Department of Chemistry } \\
\text { Wenger, Oliver; University of Basel, Department of Chemistry }\end{array}$ \\
\hline
\end{tabular}

SCHOLARONE

Manuscripts 


\title{
Charge Delocalization in an Organic Mixed Valent Bithiophene is Greater than in a Structurally
}

\section{Analogous Biselenophene}

\author{
Ann Christin Jahnke, ${ }^{\dagger}$ Jonny Proppe, ${ }^{\star \xi}$ Mariana Spulber, ${ }^{\dagger}$ Cornelia G. Palivan, ${ }^{\dagger}$ Carmen \\ Herrmann, ${ }^{*}+$ and Oliver S. Wenger ${ }^{*}+$ \\ ${ }^{\dagger}$ Department of Chemistry, University of Basel, St. Johanns-Ring 19, CH-4056 Basel, \\ Switzerland \\ $\$$ Institute of Inorganic and Applied Chemistry, University of Hamburg, Martin-Luther-King- \\ Platz 6, D-20146 Hamburg, Germany \\ $\S$ Present address: Laboratory of Physical Chemistry, ETH Zurich, Vladimir-Prelog-Weg 2, CH- \\ 8093 Zurich, Switzerland \\ carmen.herrmann@chemie.uni-hamburg.de; oliver.wenger@unibas.ch
}




\begin{abstract}
A series of selenophenes with redox-active amine end-capping groups was synthesized and investigated. A combination of cyclic voltammetry, optical absorption, EPR spectroscopy and quantum-chemical calculations based on Kohn-Sham density functional theory was used to explore charge delocalization in the monocationic mixed-valence forms of these selenophenes, and the results were compared to those obtained from analogous studies of structurally identical thiophenes. The striking finding is that the comproportionation constant $\left(\mathrm{K}_{\mathrm{c}}\right)$ for the experimentally investigated biselenophene is more than two orders of magnitude lower than for its bithiophene counterpart (in $\mathrm{CH}_{3} \mathrm{CN}$ with $0.1 \mathrm{M} \mathrm{TBAPF}_{6}$ ), and the electronic coupling between the two amine end-capping groups in the mixed-valent biselenophene monocation is only roughly half as strong as in the corresponding bithiophene monocation. These are surprisingly large differences given the structural similarity between the respective biselenophene and bithiophene molecules. However, the computationally determined comproportionation constants for biselenophene and bithiophene are almost identical, and the electronic coupling in the monocationic biselenophene is only slightly smaller than that in the monocationic bithiophene. We assume that the external electric field may be responsible for the differences in monocation stabilities between experiment and computation. Our findings indicate that charge delocalization across individual selenophenes tends to be less pronounced than across individual thiophenes, and this may have important implications for long-range charge transfer across selenophene oligomers or polymers.
\end{abstract}




\section{KEYWORDS}

Mixed valence, cyclic voltammetry, optical absorption spectroscopy, electron paramagnetic resonance, electronic coupling

\section{INTRODUCTION}

Conducting oligomers and polymers have found use in many different applications including for example photovoltaic cells or light-emitting diodes. ${ }^{1,2}$ Polythiophene is one of the most investigated conducting polymers. ${ }^{3}$ Recently there has been increasing interest in polymers of other chalcogenophenes, that is, in polyfuranes, ${ }^{4,5}$ polyselenophenes, ${ }^{6-8}$ and polytellurophenes. ${ }^{9-11}$ Whether or not selenophenes might be even better building blocks for the formation of charge conducting polymers than thiophenes is an intriguing question. In this context it seemed useful to explore to what extent charge can be delocalized over individual chalcogenophene monomer building blocks or over short oligomers thereof. One fairly simple possibility to do this is to attach redox-active groups to the two ends of the molecules of interest and to oxidize (or reduce) one of these end-capping groups in order to generate mixed-valence species. ${ }^{12-15}$ This experimental approach has been applied numerous times to thiophene derivatives, ${ }^{14,16-25}$ but selenophenes are comparatively poorly explored in this regard. ${ }^{26} \mathrm{We}$ have recently reported the first comparative study of charge delocalization across the entire chalcogenophene series ranging from furan to tellurophene. ${ }^{27}$

As redox-active units for mixed-valence studies ferrocenes, ${ }^{5,28}$ ruthenium complexes, ${ }^{29,30}$ and triarylamines are particularly popular choices. ${ }^{12,13,31}$ We have chosen the latter because 
bis(triarylamine) monocations tend to give intervalence absorption bands which are readily detectable. In Scheme 1 the amine end-capped selenophenes which were investigated in the present study $(\mathbf{1}, \mathbf{2}, \mathbf{3}, \mathbf{4})$ are shown along with thiophene analogs (I, II, IV) which we have previously explored. $^{23}$

Scheme 1. Chemical structures of the amino-decorated selenophenes investigated in this work (1 -4) and of their thiophene analogs which we recently explored $(\mathbf{I}-\mathbf{I V}) .^{23}$
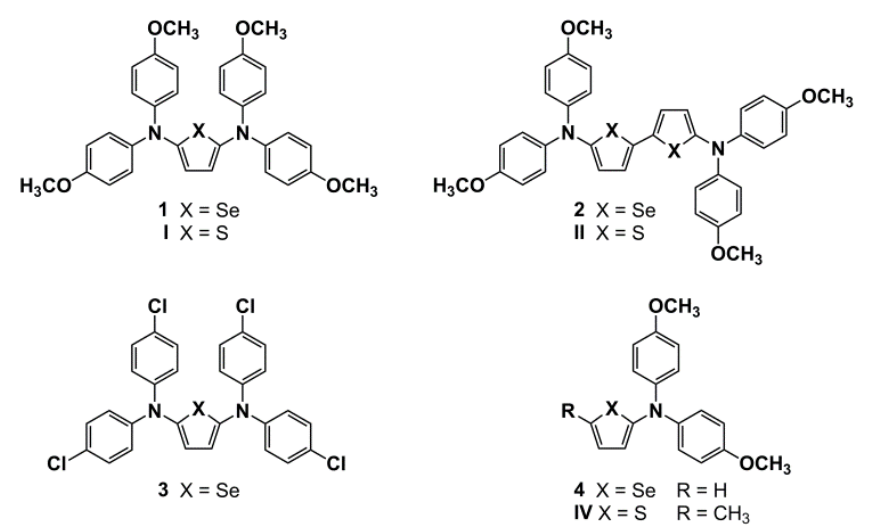

Selenophenes $\mathbf{1}-\mathbf{3}$ were investigated by a combination of cyclic voltammetry, optical absorption, and EPR spectroscopy. Our study reveals certain analogies but also some remarkable differences between the selenophenes and the corresponding thiophenes.

\section{RESULTS AND DISCUSSION}

Synthesis. Compounds $\mathbf{1}-\mathbf{4}$ were obtained using a palladium(0)-catalyzed C,N-cross coupling reaction between one equivalent of 2,5-dibromoselenophene or 5,5'-dibromo-2,2'-biselenophene and two equivalents of secondary amine. For compound 4, 2-bromoselenophene was reacted 
with one equivalent of secondary amine. Our attempts to isolate a terselenophene analog to $\mathbf{1}$ and 2 were unsuccessful because of purification problems of the final product.

All compounds were purified by column chromatography on silica gel, and they were characterized by high-resolution mass spectrometry, NMR spectroscopy, and by elemental analysis. Detailed synthetic protocols and product characterization data for all new compounds are in the Supporting Information.

Computational methodology. Oxidation potentials: Molecular and electronic structures of neutral, monocationic and bicationic forms of 1, 2, 3, I and II were optimized employing KohnSham density-functional theory (KS-DFT) combined with a protocol by Renz and Kaupp developed for the prediction of electronic communication in organic mixed-valence compounds. $^{32-34}$ This protocol includes the use of the BLYP35 hybrid functional (35\% exactexchange admixture) ${ }^{32,35-37}$ of Ahlrichs' def-TZVP basis set ${ }^{38}$ and of the conductor-like polarizable continuum model (CPCM) to consider solvent effects. ${ }^{39,40}$ We chose the dielectric constant of acetonitrile because this was the solvent in the experimental investigations. The BMK hybrid functional ${ }^{41}$ (42\% exact-exchange admixture) and the BLYP80 ${ }^{35-37}$ hybrid functional ( $80 \%$ exact-exchange admixture) were additionally employed. Furthermore, singlepoint calculations were performed on the monocations with the optimized neutral molecular structure and on the bications with the optimized monocationic molecular structure. For further details and the choice of initial structures, see the Supporting Information. The term "ionization potential" might be technically more correct for our calculated values but we will use the term "oxidation potential" throughout this manuscript to make the relationship between experiment and calculation more evident. 
Optical absorption spectra: Optimized (BLYP35/TZVP/CPCM:MeCN) molecular structures of neutral and monocationic forms of 1, 2, 3, I and II were used as input to calculate the three lowest-energy excitations employing time-dependent density-functional theory (TD-DFT) combined with the mentioned protocol by Renz and Kaupp. We chose the dielectric constants of acetonitrile and dichloromethane because these two solvents were used for the experimental UVVis-NIR studies. The BMK hybrid functional and the M06HF hybrid functional (100\% exactexchange admixture) were additionally employed. ${ }^{42,43}$

Cyclic voltammetry. Figure 1 shows cyclic voltammograms of compounds 1 (a), 2 (b), and 3 (c) in dry and deoxygenated acetonitrile measured in the presence of $0.1 \mathrm{M}$ tetrabutylammonium hexafluorophosphate $\left(\mathrm{TBAPF}_{6}\right)$ electrolyte at scan rates of $100 \mathrm{mV} / \mathrm{s}$. The waves at $-0.51 \mathrm{~V}$ vs. $\mathrm{Fc}^{+/ 0}$ (marked by the dotted vertical line) are due to decamethylferrocene which was added for internal potential calibration, the experimental uncertainty associated with our potential measurements is on the order of $0.03 \mathrm{~V}$.

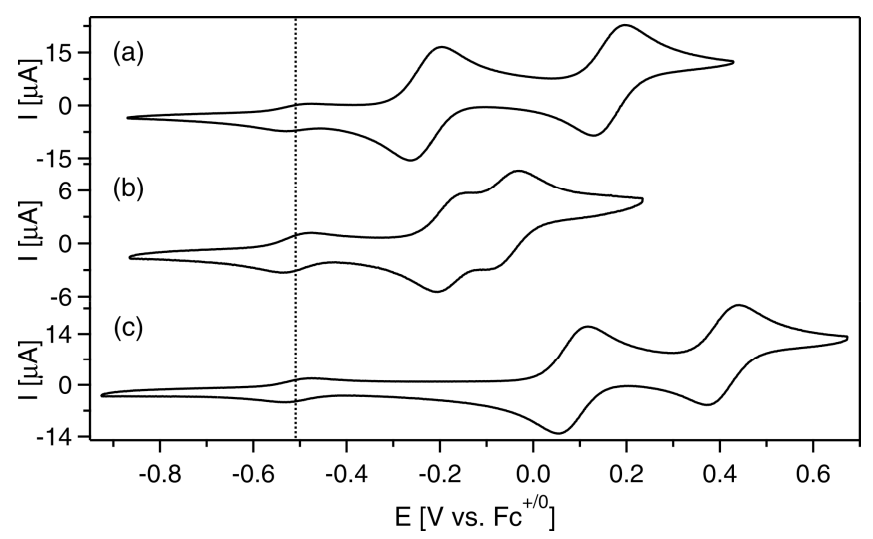

Figure 1. Cyclic voltammograms of compounds $\mathbf{1}-\mathbf{3}$ in dry and deoxygenated $\mathrm{CH}_{3} \mathrm{CN}$ measured in presence of $0.1 \mathrm{M} \mathrm{TBAPF}_{6}$ electrolyte: (a) compound 1; (b) compound 2; (c) 
compound 3. The waves at $-0.51 \mathrm{~V}$ vs. $\mathrm{Fc}^{+/ 0}$ (dashed vertical line) are due to added decamethylferrocene. The scan rate was $100 \mathrm{mV} / \mathrm{s}$.

In the voltage range shown in Figure 1 the voltammograms of all three compounds exhibit two reversible waves which are clearly separate from each other. They are attributed to one-electron oxidation of the two amine end-capping groups. For a given wave, the average separation between oxidative and reductive peak currents is $65 \mathrm{mV}$. Expectedly, reference molecule 4 exhibits only one oxidation wave in the same potential range (Figure S1 of the Supporting Information) because it contains only one amino group. Moreover, oxidation is irreversible in this case, and this is presumably due to electropolymerization phenomena. Analogous experiments with $\mathbf{1}-\mathbf{3}$ in dichloromethane produce lower quality voltammograms (Figure S2) in which for a given oxidation wave the voltage separation between oxidative and reductive peak current is substantially larger (on average $260 \mathrm{mV}$ ) than in acetonitrile. The electrochemical potentials for one- and two-electron oxidation of compounds $\mathbf{1}-\mathbf{3}$ in acetonitrile and dichloromethane are summarized in Table 1.

Table 1. Electrochemical potentials for one- and two-electron oxidation of compounds $\mathbf{1}-\mathbf{3}$ in $\mathrm{V}$ vs. $\mathrm{Fc}^{+/ 0}$ in acetonitrile and dichloromethane. The supporting electrolyte was $0.1 \mathrm{M} \mathrm{TBAPF}_{6}$.

\begin{tabular}{|c|c|c|c|c|c|c|c|c|}
\hline & \multicolumn{5}{|c|}{$\mathrm{CH}_{3} \mathrm{CN}$} & \multicolumn{4}{c|}{$\mathrm{CH}_{2} \mathrm{Cl}_{2}$} \\
\hline compd & $\mathrm{E}_{1 / 2}^{++0}$ & $\mathrm{E}_{1 / 2}{ }^{2+/+}$ & $\Delta \mathrm{E}[\mathrm{mV}]$ & $\mathrm{K}_{\mathrm{c}}$ & $\mathrm{E}_{1 / 2}^{+/ 0}$ & $\mathrm{E}_{1 / 2}{ }^{2+/+}$ & $\Delta \mathrm{E}[\mathrm{mV}]$ & $\mathrm{K}_{\mathrm{c}}$ \\
\hline $\mathbf{1}^{a}$ & -0.23 & 0.16 & 393 & $4.6 \times 10^{6}$ & -0.27 & 0.23 & 502 & $3.2 \times 10^{8}$ \\
\hline $\mathbf{2}^{a}$ & -0.17 & -0.06 & 109 & $7.0 \times 10^{1}$ & -0.21 & 0.06 & 269 & $2.5 \times 10^{4}$ \\
\hline $\mathbf{3}^{a}$ & 0.09 & 0.41 & 321 & $2.8 \times 10^{5}$ & 0.06 & 0.44 & 372 & $2.0 \times 10^{6}$ \\
\hline
\end{tabular}




\begin{tabular}{|c|c|c|c|c|c|c|c|c|}
\hline $\mathbf{I}^{b}$ & -0.23 & 0.22 & 450 & $4.2 \times 10^{7}$ & & & & \\
\hline $\mathbf{I I}^{b}$ & -0.17 & 0.08 & 250 & $1.7 \times 10^{4}$ & & & & \\
\hline
\end{tabular}
$\Delta \mathrm{E}=\mathrm{E}_{1 / 2}{ }^{+/ 0}-\mathrm{E}_{1 / 2}{ }^{2+/+} . \mathrm{K}_{\mathrm{c}}$ is the comproportionation constants as defined in the text. ${ }^{a}$ This $^{\text {Th }}$
work; ${ }^{b}$ from ref. ${ }^{23}$.

Compounds $\mathbf{1}$ and $\mathbf{2}$ only differ in the length of the selenophene bridge, resulting in a similar first oxidation potential $\left(\mathrm{E}_{1 / 2}{ }^{+/ 0}\right.$, second column of Table 1$)$ in both solvents. Compounds $\mathbf{1}$ and $\mathbf{3}$ both contain a monoselenophene bridge but differ in their amino groups. The first oxidation potential is significantly less positive for $\mathbf{1}$ than for $\mathbf{3}$ which may be caused by the higher electron density at the nitrogen atoms in $\mathbf{1}$ due to electron-donating methoxy groups. The less positive oxidation potential is confirmed by DFT calculations (Table S2 in the Supporting Information).

Of particular relevance in the context of mixed-valence phenomena are the differences $(\Delta \mathrm{E})$ between the electrochemical potentials for oxidation of the first $\left(\mathrm{E}_{1 / 2}{ }^{+/ 0}\right)$ and the second $\left(\mathrm{E}_{1 / 2}{ }^{2+/+}\right)$ redox center (fourth column of Table 1). On the basis of these $\Delta \mathrm{E}$ values the comproportionation constants $\left(\mathrm{K}_{\mathrm{c}}=10^{(\Delta \mathrm{E} / 59 \mathrm{mV})}\right)$ can be estimated (fifth column of Table 1). $\mathrm{K}_{\mathrm{c}}$ is a measure of the stability of the monocationic (mixed-valent) state of compounds $\mathbf{1}-\mathbf{3}^{44}$ The comproportionation constant is often largely determined by electrostatic effects, and it can be tricky to extract meaningful information on electronic coupling matrix elements on the basis of electrochemical data. ${ }^{45}$ In acetonitrile, $K_{c}$ decreases by roughly 5 orders of magnitude between compounds $\mathbf{1}$ and 2, but the $K_{c}$ values of compounds 1 and $\mathbf{3}$ differ by only a factor of 16. Expectedly, an increase in bridge length has a far greater influence on $\mathrm{K}_{\mathrm{c}}$ than a change in electronic structure at the redox-active unit.

Comparison with the $\mathrm{K}_{\mathrm{c}}$ values of the thiophene analogs $\mathbf{I}$ and II is particularly interesting. Direct comparison is possible in this case because I and II were investigated under precisely the 
same conditions, ${ }^{23}$ i. e., using the same solvent and the same electrolyte hence solvation and ion pairing effects are expected to be similar in both studies. ${ }^{28}$ For monothiophene compound $\mathbf{I}$ in $\mathrm{CH}_{3} \mathrm{CN}$ with $\mathrm{TBAPF}_{6}$ we found $\mathrm{K}_{\mathrm{c}}=4.2 \cdot 10^{7}$ (Table 1) which is nearly an order of magnitude greater than the $K_{c}$ value obtained for the monoselenophene compound $\mathbf{1}\left(4.6 \cdot 10^{6}\right.$, Table 1$)$. For bithiophene compound II we found $\mathrm{K}_{\mathrm{c}}=1.7 \cdot 10^{4}$ which is a factor of 240 greater than the $\mathrm{K}_{\mathrm{c}}$ value (70) obtained for biselenophene compound 2.

The key finding from the cyclic voltammetry studies is that the comproportionation constants for selenophenes $\mathbf{1}$ and $\mathbf{2}$ are substantially lower than those of the corresponding thiophenes $\mathbf{I}$ and II. Moreover, $\mathrm{K}_{\mathrm{c}}$ decreases significantly more when going from the monoselenophene (1) to the biselenophene (2) (a factor of 265000 ) than when going from the monothiophene (I) to the bithiophene (II) (a factor of 2500). The lower $\mathrm{K}_{\mathrm{c}}$ values in the selenophenes (and their more pronounced distance-dependence) may be a manifestation of weaker (through-bond) electronic communication across mono- and biselenophene than across mono- and bithiophene. However, it may also be important to consider electrostatic effects even though the distances between the redox moieties are almost the same in both cases. Moreover, since organic mixed-valence compounds are known to exhibit a significant fraction of spin on the bridge, the external electric field might influence the two bichalcogenophenes to a different extent because of the larger polarizability of selenium compared to sulfur (vide infra).

Optical absorption spectroscopy. The black traces in Figure 2 are the optical absorption spectra of compounds $\mathbf{1}-\mathbf{3}$ in acetonitrile at room temperature. None of the three molecules shows any significant absorption below $17000 \mathrm{~cm}^{-1}$ in the charge-neutral form. The colored traces were measured after addition of increasing amounts of $\mathrm{Cu}\left(\mathrm{ClO}_{4}\right)_{2}$ which led to chemical 
oxidation of the selenophene compounds. In Figure 2 we show the spectra which were detected after addition of 0.5 (purple), 1.0 (green), 1.5 (orange), and 2.0 equivalents (red) of $\mathrm{Cu}\left(\mathrm{ClO}_{4}\right)_{2}$ to $4.0 \cdot 10^{-5} \mathrm{M}$ solutions of the selenophenes.

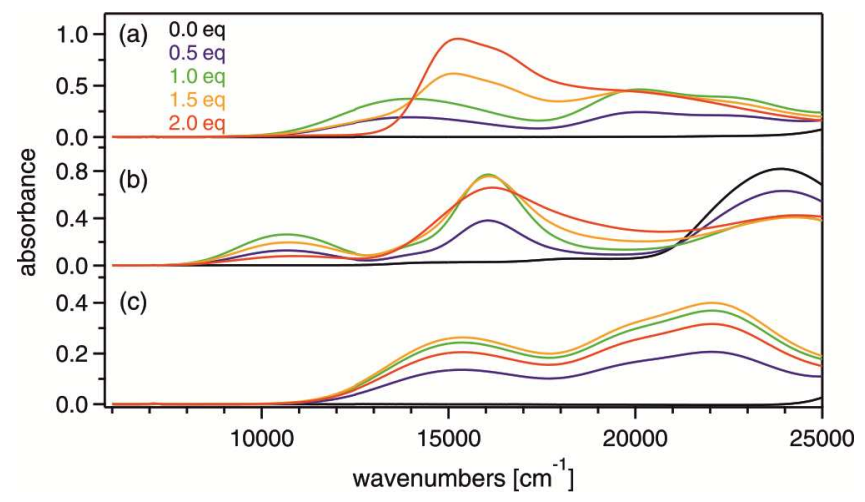

Figure 2. Optical absorption spectra of $4.0 \cdot 10^{-5} \mathrm{M}$ solutions of (a) selenophene 1, (b) selenophene 2, and (c) selenophene 3 in $\mathrm{CH}_{3} \mathrm{CN}$ (black traces). The colored traces were measured after addition of $\mathrm{Cu}\left(\mathrm{ClO}_{4}\right)_{2}$ as a chemical oxidant.

Initially, addition of $\mathrm{Cu}\left(\mathrm{ClO}_{4}\right)_{2}$ oxidant to solutions containing mono- (1) and biselenophene (2) leads to new absorption bands at $\sim 14000 \mathrm{~cm}^{-1}$ and $\sim 10700 \mathrm{~cm}^{-1}$ (blue traces in Figure 2a/b). These bands reach maximal absorbance after addition of 1 equivalent of oxidant (green traces) and then decrease upon further addition of $\mathrm{Cu}\left(\mathrm{ClO}_{4}\right)_{2}$ (orange and red traces). In other words, these low-energy bands are observed when substantial concentrations of the one-electron oxidized species $\mathbf{1}^{+}$and $\mathbf{2}^{+}$are present. Qualitatively, the spectra of $\mathbf{1}^{+}$and $\mathbf{2}^{+}$are very similar to those of $\mathbf{I}^{+}$and $\mathbf{I I}^{+}$which were measured previously under identical conditions. ${ }^{23}$ Upon oxidation of the bis(4-chlorophenyl)amino-decorated selenophene (3) there is a new band at $\sim 15200 \mathrm{~cm}^{-1}$ (blue trace in Figure 2c) which we interpret as the analog to the $14000 \mathrm{~cm}^{-1}$ band of $\mathbf{1}^{+}$(Figure 
2a). Replacement of methoxy groups on the amines by chloro-substituents thus induces a blueshift of the lowest energetic monocation absorption. As a consequence, the lowest-energetic absorption of $3^{+}$comes energetically close to electronic transitions of different origin (and possibly due to different species such as for example $\mathbf{3}^{\mathbf{2 +}}$ ), and this might explain why even after addition of 2 equivalents of $\mathrm{Cu}\left(\mathrm{ClO}_{4}\right)_{2}$ there is still significant absorbance around $15000 \mathrm{~cm}^{-1}$ in the particular case of Figure 2c (red trace). Quantum-chemical calculations (BLYP35/TZVP/CPCM:MeCN) support this hypothesis (see Table S9). Moreover, the substantially higher oxidation potential of $\mathbf{3}^{+}$(compared to $\mathbf{1}^{+}$and $\mathbf{2}^{+}$) makes the second oxidation with $\mathrm{Cu}$ (II) less favorable in this case. There is no indication for the formation of degradation products on the timescale on which these UV-Vis studies were performed.

The lowest-energy absorption bands of bis(triarylamine) monocations are commonly interpreted as intervalence (or charge resonance) absorption bands. ${ }^{12,13,22,46}$ The fact that such bands are observed is an indication of significant electronic interaction between the individual redox centers. In the following we perform a quantitative analysis of the intervalence absorptions detected in Figure 2.

The black traces in Figure 3 are the experimental absorption spectra of $\mathbf{1}^{+}$(a), $\mathbf{2}^{+}$(b), and $\mathbf{3}^{+}$(c) in $\mathrm{CH}_{3} \mathrm{CN}$ at room temperature after addition of 1.0 eq of $\mathrm{Cu}\left(\mathrm{ClO}_{4}\right)_{2}$ to $4.0 \cdot 10^{-5} \mathrm{M}$ solutions of the charge-neutral compounds. ${ }^{47}$ The colored traces in Figure 3 are Gaussian fits to the experimental data. The Gaussians marked in green were used to fit the low-energy intervalence absorption bands, whereas the Gaussians represented by dashed purple traces were necessary to fit the higher-energy portions of the experimental spectrum. The latter are of no further interest. The dotted red traces represent the sums of the individual (green and purple) Gaussians. They match the experimental (black) traces nearly perfectly. 


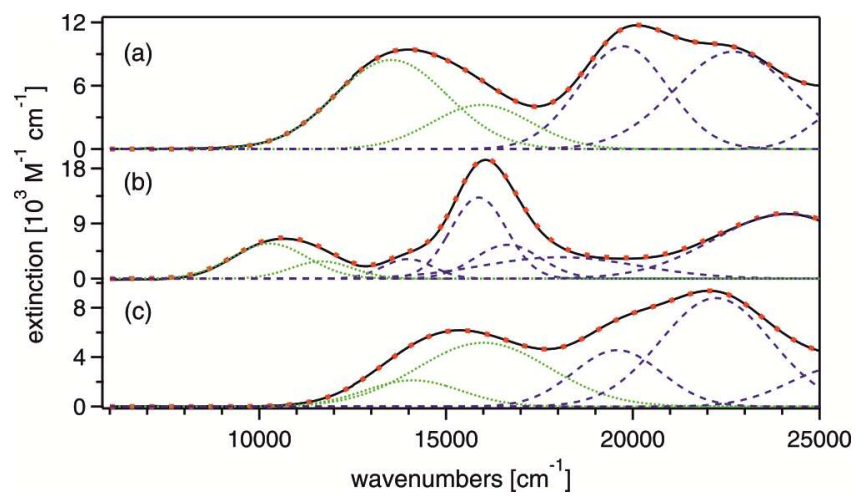

Figure 3. Black traces: Experimental absorption spectra of $\mathbf{1}^{+}(\mathrm{a}), \mathbf{2}^{+}$(b), and $\mathbf{3}^{+}$(c) in $\mathrm{CH}_{3} \mathrm{CN}$. Blue and green traces: Gaussian fits to the experimental data. Dotted red traces: Sums of the individual Gaussian fit functions. The parameters from the Gaussian functions used to fit the intervalence absorption bands (dotted green lines) are listed in Table 2.

Table 2. Parameters obtained from analysis of the intervalence absorptions in Figure 3.

\begin{tabular}{|c|c|c|c|c|c|c|c|c|}
\hline compd & $\begin{array}{c}v_{1 / 2[\mathrm{high}]} \\
{\left[\mathrm{cm}^{-1}\right]}\end{array}$ & $\begin{array}{c}v_{1 / 2[\text { low }]} \\
{\left[\mathrm{cm}^{-1}\right]}\end{array}$ & $v_{1 / 2[\mathrm{high}]} / v_{1 / 2[\mathrm{low}]}$ & $\begin{array}{c}v_{\text {max }, \mathrm{G} 1} \\
{\left[\mathrm{~cm}^{-1}\right]}\end{array}$ & $\begin{array}{c}v_{\text {max, G2 }} \\
{\left[\mathrm{cm}^{-1}\right]}\end{array}$ & $\begin{array}{c}v_{1 / 2, \mathrm{G} 1} \\
{\left[\mathrm{~cm}^{-1}\right]}\end{array}$ & $\begin{array}{c}v_{1 / 2, \mathrm{G} 2} \\
{\left[\mathrm{~cm}^{-1}\right]}\end{array}$ & $\begin{array}{c}v_{1 / 2, \text { class II }} \\
{\left[\mathrm{cm}^{-1}\right]}\end{array}$ \\
\hline $\mathbf{1}^{+}$ & 5605 & 4055 & 1.38 & 13535 & 15955 & 3520 & 3110 & 5675 \\
\hline $\mathbf{2}^{+}$ & 3130 & 2905 & 1.08 & 10275 & 11670 & 2390 & 1950 & 4965 \\
\hline $\mathbf{3}^{+}$ & 4880 & 4420 & 1.10 & 14110 & 16005 & 3035 & 4170 & 5960 \\
\hline
\end{tabular}

$v_{1 / 2[\mathrm{high}]}$ and $v_{1 / 2[\mathrm{low}]}$ are defined in the text. $v_{\max , \mathrm{Gi}}$ is the energetic position of the maximum of the i-th Gaussian (dotted green traces in Figure 3). $v_{1 / 2, \mathrm{Gi}}$ is the full width at half-maximum

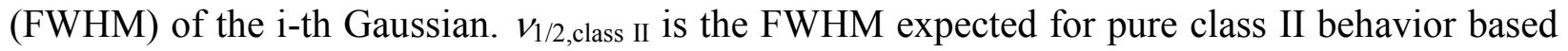
on equation 1.

The IVCT bands of all three monocations $\left(\mathbf{1}^{+}, \mathbf{2}^{+}, \mathbf{3}^{+}\right)$can only be fitted satisfactorily when using two Gaussians. Based on Hush theory, so-called class II mixed-valence species, i. e., those exhibiting only partial charge delocalization, ${ }^{48}$ are expected to exhibit Gaussian-shaped IVCT 
bands. ${ }^{49-52}$ The finding that the low-energy bands of $\mathbf{1}^{+}, \mathbf{2}^{+}$, and $\mathbf{3}^{+}$cannot be fitted adequately with single Gaussians can therefore be interpreted as a deviation from pure class II behavior. Completely delocalized class III systems are known to exhibit strongly asymmetrical IVCT bands, ${ }^{51}$ but in our case the asymmetry is not very pronounced, and it seems plausible that $\mathbf{1}^{+}, \mathbf{2}^{+}$, and $\mathbf{3}^{+}$are in fact borderline class II / class III systems like numerous other bis(triarylamine) monocations. $^{12,13,46}$ A simple measure of the asymmetry is the ratio $v_{1 / 2[\mathrm{high}]} / v_{1 / 2[\mathrm{low}]}$ (fourth column of Table 2). $v_{1 / 2[\text { high] }}$ is twice the bandwidth on the high-energy side of the IVCT band (second column of Table 2) and $v_{1 / 2[\text { low }]}$ is twice the bandwidth on the low-energy side of the IVCT band (third column of Table 2). The $v_{1 / 2[\text { high }]} / v_{1 / 2[\text { low }]}$ ratios vary between 1.08 and $1.38, \mathrm{i}$. e., the asymmetry is not very pronounced as noted above.

For the IVCT bands of class II mixed-valence species, one expects a relation between IVCT bandwidth ( $\left.v_{1 / 2 \text {,class II }}\right)$ and energetic position of the IVCT band maximum $\left(v_{\max }\right)$ as described by equation $1 .^{51} v_{\max }$ was extracted directly from the experimental absorption spectra in Figure 3 (maximum of the lowest-energy band).

$$
v_{1 / 2, \text { class II }}=\sqrt{2310 \cdot v_{\max }}
$$

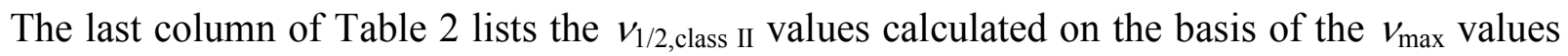
extracted from the experimental spectra in Figure 3. The bandwidths calculated in this manner for $\mathbf{1}^{+}, \mathbf{2}^{+}$, and $\mathbf{3}^{+}$are all significantly broader than the experimentally observed bandwidths. For instance, for $1^{+}$one calculates $v_{1 / 2, \text { class II }}=5675 \mathrm{~cm}^{-1}$, whereas the experimental value $\left(\left(v_{1 / 2[\mathrm{high}]}+\right.\right.$

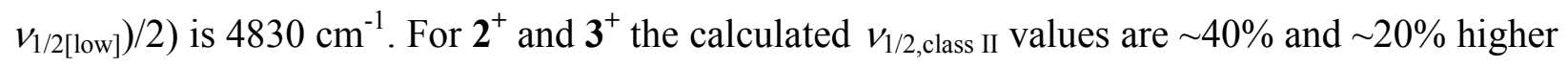
than the experimental bandwidths. These sizeable discrepancies between calculated $v_{1 / 2, \text { class II }}$ 
values and experimental bandwidths can be interpreted as an additional indication for a deviation from pure class II behavior.

The solvent dependence of the IVCT bands is non-negligible. Figure 4 displays absorption spectra of $\mathbf{1}^{+}(\mathrm{a}), \mathbf{2}^{+}(\mathrm{b})$, and $\mathbf{3}^{+}$(c) in acetonitrile (black traces) and dichloromethane (red traces). This change in solvent causes shifts of the IVCT band maxima by $1030 \mathrm{~cm}^{-1}\left(\mathbf{1}^{+}\right), 250 \mathrm{~cm}^{-1}\left(\mathbf{2}^{+}\right)$, and $850 \mathrm{~cm}^{-1}\left(3^{+}\right)$. Poor solubility precluded measurements in less polar solvents. The IVCT bands of completely delocalized class III mixed-valence compounds tend to exhibit weaker solvent dependencies than those observed here.

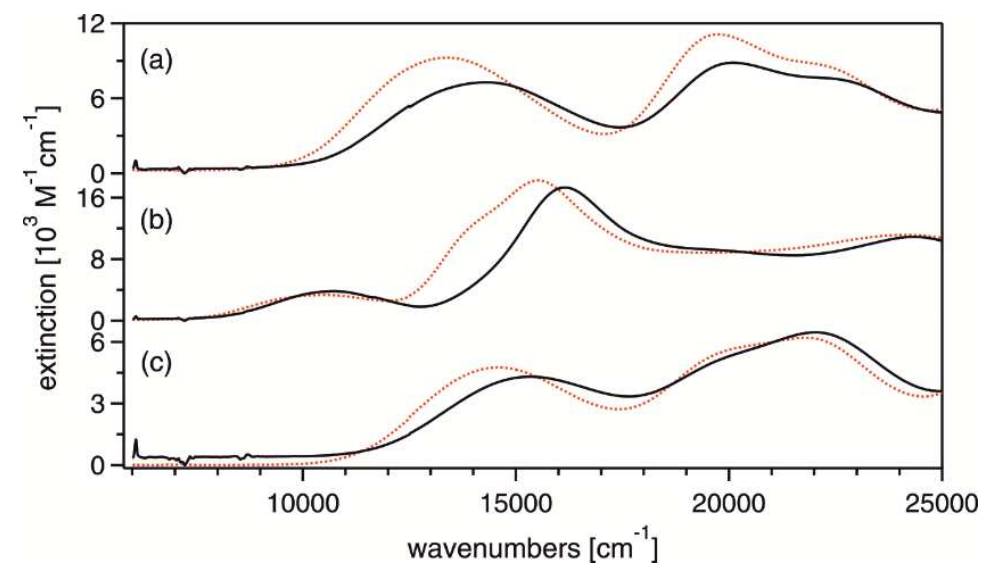

Figure 4. Optical absorption spectra of (a) $\mathbf{1}^{+}$, (b) $\mathbf{2}^{+}$, and (c) $\mathbf{3}^{+}$in acetonitrile (black traces) and in dichloromethane (red dotted traces).

The conclusion from this section is that clear-cut assignment of $\mathbf{1}^{+}, \mathbf{2}^{+}, \mathbf{3}^{+}$to either class II or class III is difficult. Some of the experimental evidence is more compatible with class III (asymmetry and relatively narrow widths of IVCT bands) whereas other evidence points towards class II (solvent dependence). The systems considered here most likely belong to a family of 
compounds named borderline class II / class III systems. ${ }^{46,51,52}$ For the thiophene analogs $\mathbf{I}^{+}, \mathbf{I I}^{+}$, and $\mathrm{III}^{+}$we have previously arrived at the same conclusion. ${ }^{23}$

Determination of electronic coupling matrix elements. The matrix element quantifying the electronic coupling between individual redox centers $\left(H_{A B}\right)$ is in relation to the transition dipole moment $\left(\mu_{\mathrm{ge}}\right)$ associated with the IVCT as described by equation $2 .^{22,46,49}$

$H_{A B}=\frac{\mu_{\mathrm{ge}} \cdot v_{\max }}{e \cdot R}$ (eq. 2)

$v_{\max }$ is the energetic position of the IVCT band maximum (see above), $R$ is the effective charge transfer distance, and $e$ is the elemental charge. The transition dipole moment in units of $e \cdot \AA$ can be determined from integration of the IVCT band as described by equation $3 .^{22,46,49}$

$$
\mu_{\mathrm{ge}}=0.09584 \cdot \sqrt{\frac{\int \varepsilon(v) \cdot \mathrm{d} v}{v_{\max }}}
$$

The $\mu_{\mathrm{ge}}$ values reported in the fifth column of Table 3 were determined on the basis of the two Gaussian functions needed to fit the experimental IVCT bands (dotted green traces in Figure 3). Equation 3 assumes that the IVCT spectra are properly represented in the form of extinction coefficient (in $\mathrm{M}^{-1} \mathrm{~cm}^{-1}$ ) versus wavenumber (in $\mathrm{cm}^{-1}$ ).

Table 3. Estimation of electronic coupling matrix elements ( $H_{A B}$ values).

\begin{tabular}{|l|l|l|l|l|l|}
\hline compd & $d_{\mathrm{NN}}[\AA]$ & $v_{\max }\left[\mathrm{cm}^{-1}\right]$ & $\mu_{\mathrm{ge}}[\mathrm{D}]$ & $\mu_{\mathrm{ge}}[e \cdot \AA]$ & $H_{A B}\left[\mathrm{~cm}^{-1}\right]$ \\
\hline
\end{tabular}




\begin{tabular}{|c|c|c|c|c|c|c|}
\hline & & & & & $R=d_{\mathrm{NN}}$ & $R=2 / 3 \cdot d_{\mathrm{NN}}$ \\
\hline $\mathbf{1}^{+a}$ & 5.2 & 13950 & 5.5 & 1.1 & 3080 & 4620 \\
\hline $\mathbf{2}^{+a}$ & 9.1 & 10660 & 4.2 & 0.9 & 1010 & 1520 \\
\hline $\mathbf{3}^{+a}$ & 5.2 & 15385 & 4.2 & 0.9 & 2620 & 3920 \\
\hline $\mathbf{I}^{+b}$ & 5.2 & 13850 & 5.7 & 1.2 & 3160 & 4790 \\
\hline $\mathbf{I I}^{+b}$ & 9.1 & 10620 & 7.7 & 1.6 & 1870 & 2835 \\
\hline
\end{tabular}

${ }^{a}$ This work; ${ }^{b}$ From ref. ${ }^{23} \cdot d_{\mathrm{NN}}$ is the N-N distance estimated from molecular modeling, $v_{\max }$ is the energetic position of the IVCT absorption band maximum, $\mu_{\mathrm{ge}}$ is the transition dipole moment associated with the IVCT.

The trickiest part in evaluating $H_{A B}$ on the basis of equations 2 and 3 is the determination of the effective electron transfer distance. Many prior examples have shown that $R$ need not correspond to the geometrical distance between redox centers. ${ }^{22,53-55}$ Moreover, in organic mixed-valence compounds the redox activity usually cannot be pinpointed to a single atom hence it is inherently difficult to determine $R$. One possibility is to use Stark spectroscopy, another is to perform calculations. $^{22,56,57}$ For our purposes it seems sufficient to consider two limiting cases: The upper limit of $R$ is taken as the geometrical distance between nitrogen atoms $\left(d_{\mathrm{NN}}\right)$. For the lower limit we assume that $R=2 / 3 \cdot d_{\mathrm{NN}}$ based on prior studies of comparable organic mixed-valence compounds. ${ }^{12,13,23,53}$ The resulting electronic coupling matrix elements based on these two limiting assumptions are reported in the last two columns of Table 3.

The first thing we note is that $H_{A B}$ for monoselenophene $\mathbf{1}^{+}$and monothiophene $\mathbf{I}^{+}$are very similar, regardless whether the $R=d_{\mathrm{NN}}$ or the $R=2 / 3 \cdot d_{\mathrm{NN}}$ limit is considered. In the latter limit, $H_{A B}=4620 \mathrm{~cm}^{-1}$ for $\mathbf{1}^{+}$and $H_{A B}=4790 \mathrm{~cm}^{-1}$ for $\mathbf{I}^{+}$. By contrast, biselenophene $\mathbf{2}^{+}$exhibits $H_{A B}$ values which are roughly $45 \%$ lower (in both limits) compared to the electronic coupling matrix element of bithiophene $\mathbf{I I}^{+}$. In other words, $H_{A B}$ decreases much more strongly between $\mathbf{1}^{+}$and $\mathbf{2}^{+}$ 
than between $\mathbf{I}^{+}$and $\mathbf{I I}^{+}$. This is in line with the much stronger decrease in $\mathrm{K}_{\mathrm{c}}$ between $\mathbf{1}$ and $\mathbf{2}$ (factor of $\sim 65^{\prime} 000$ ) than between I and II (factor of $\sim 2500$ ), see above.

According to superexchange theory, ${ }^{58}$ electronic coupling matrix elements exhibit an exponential distance dependence following equation $4 .{ }^{59}$

$$
H_{A B}(R)=H_{A B}^{(0)} \cdot \exp \left(-\frac{1}{2} \cdot \beta \cdot\left(R-R^{(0)}\right)\right)
$$

Here, $H_{A B}{ }^{(0)}$ is the electronic coupling matrix element at a reference distance $R^{(0)}$, and $\beta$ is the so-called distance decay constant. The factor of $1 / 2$ is used to make the $\beta$ values from equation 4 directly comparable to the distance decay constants extracted from studies of electron transfer rates (which are proportional to $H_{A B}{ }^{2}$ ). On the basis of the $H_{A B}$ values for $\mathbf{1}^{+}$and $\mathbf{2}^{+}$in Table 3 we find $\beta=0.57 \AA^{-1}$ in the $R=d_{\mathrm{NN}}$ limit and $\beta=0.85 \AA^{-1}$ in the $R=2 / 3 \cdot d_{\mathrm{NN}}$ limit (third row of Table 4). For $\mathbf{I}^{+}$and $\mathbf{I I}^{+}$we find $\beta=0.27 \AA^{-1}$ and $\beta=0.40 \AA^{-1}$, respectively (bottom row of Table 4). Alternatively, the use of dimensionless distance decay constants $\left(\beta_{\mathrm{n}}\right)$, characterizing the decrease of $H_{A B}$ per $\sigma$-bond between the redox active centers (here taken as the two $\mathrm{N}$-atoms), is customary. ${ }^{13}$ We obtain $\beta_{\mathrm{n}}=0.74$ for the selenophenes and $\beta_{\mathrm{n}}=0.35$ for the thiophenes (last column of Table 4). Derivations of the equations used for determining $\beta$ and $\beta_{\mathrm{n}}$ are found in the Supporting Information.

Table 4. Distance decay constants for the electronic coupling matrix elements in the selenophenes and thiophenes from Scheme 1.

\begin{tabular}{|l|c|c|}
\hline & $\beta\left[\AA^{-1}\right]$ & $\beta_{\mathrm{n}}$ \\
\hline
\end{tabular}




\begin{tabular}{|c|c|c|c|}
\hline compounds & $R=d_{\mathrm{NN}}$ & $R=2 / 3 \cdot d_{\mathrm{NN}}$ & \\
\hline $\mathbf{1}^{+} \rightarrow \mathbf{2}^{+a}$ & 0.57 & 0.85 & 0.74 \\
\hline $\mathbf{I}^{+} \rightarrow \mathbf{I I}^{+b}$ & 0.27 & 0.40 & 0.35 \\
\hline
\end{tabular}

${ }^{a}$ This work; ${ }^{b}$ From ref. ${ }^{23} . \beta$ is the distance decay constant as defined in equation $4 . \beta_{\mathrm{n}}$ is the dimensionless decay constant characterizing the decrease of $H_{A B}$ per $\sigma$-bond between the two nitrogen atoms.

It is clear that the determination of $\beta$ and $\beta_{\mathrm{n}}$ is preferably based on more than two $H_{A B}$ values, but this is not possible in the present case (see synthesis section). The $\beta$ and $\beta_{\mathrm{n}}$ values in Table 4 nevertheless capture an important difference between the selenophenes and thiophenes from Scheme 1, but extrapolation to longer oligoselenophenes and oligothiophenes should be made with caution. The key finding from this section is that the exponential drop-off in $H_{A B}$ between the mono- and biselenophene is more than twice as pronounced as for structurally analogous mono- and bithiophenes.

To put our distance decay constants from Table 4 into somewhat broader perspective we note that $\beta$ values for oligo- $p$-phenylene bridges are usually in the range from $0.4 \AA^{-1}$ to $0.8 \AA^{-1}$, depending on the attached redox-active units. ${ }^{60-62}$ For a series of phenylene-bridged bis(triarylamines) $\beta_{\mathrm{n}}=0.32$ was found. ${ }^{13,46} \mathrm{~A}$ recent study of ferrocene-decorated oligofuranes reported $\beta_{\mathrm{n}}=0.13 \AA^{-1}{ }^{5}$ Note that $\beta$ is not a strictly bridge-specific property. A given molecular bridge can produce drastically different $\beta$ values depending on the nature of the attached redoxactive units. $^{63,64}$

The influence of the redox-active moiety on $H_{A B}$ is illustrated by the comparison of monoselenophenes $\mathbf{1}^{+}$and $\mathbf{3}^{+}$. The less electron-donating nature of the chloro-substituted amine in $3^{+}$causes a non-negligible decrease of $H_{A B}$ (roughly $15 \%$ ). This is a well-known effect, ${ }^{65}$ and 
$H_{A B}$ is expected by theory to depend on the so-called tunneling-energy gap which is a function of the donor and bridge redox potentials. ${ }^{58,64}$

EPR Spectroscopy. Additional insight into the delocalization of the unpaired electron in the one-electron oxidized forms of the compounds from Scheme 1 can be gained from EPR spectroscopy. The solid lines in Figure 5 represent X-band EPR spectra of $\sim 10^{-3} \mathrm{M}$ solutions of $\mathbf{1}^{+}(\mathrm{a}), \mathbf{2}^{+}$(b) and $\mathbf{3}^{+}$(c) in acetonitrile. The radical cations were generated by chemical oxidation using $\mathrm{Cu}\left(\mathrm{ClO}_{4}\right)_{2}$ as described above. 

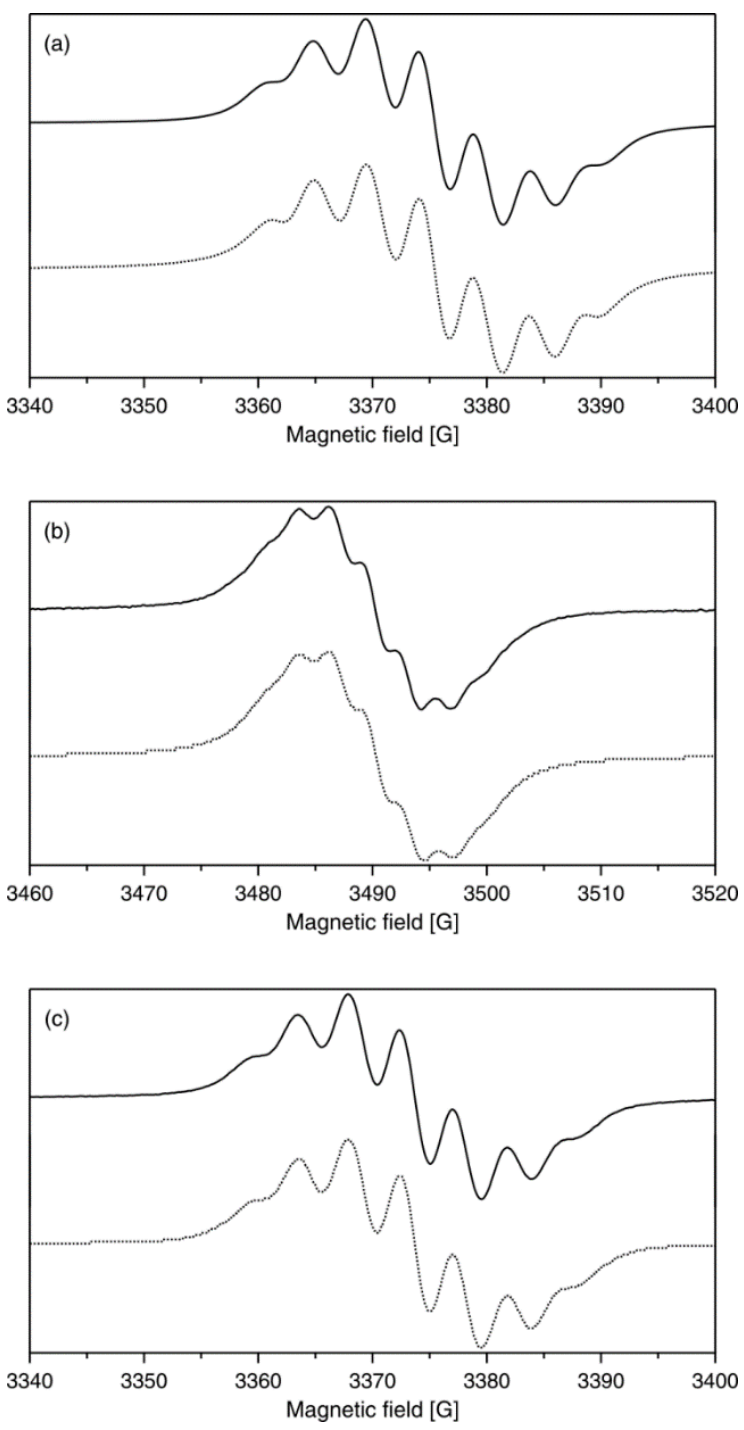

Figure 5. Solid traces: Experimental X-band EPR spectra of selenophene bridged monocations (a) $\mathbf{1}^{+}$, (b) $\mathbf{2}^{+}$, and (c) $\mathbf{3}^{+}$in $\sim 10^{-3} \mathrm{M}$ acetonitrile solution at room temperature. Dashed traces: simulated EPR spectra using the EPR parameters given in Table 5. The simulations were performed using the WinSim 2012 software.

For all three radical monocations the EPR spectra are centered at values of the gyromagnetic factor $g$ of 2.003-2.035, which is characteristic for triarylamine radical cations (Figure 5). ${ }^{22}$ The hyperfine structure of all three radical cations is due to the interaction of the unpaired electron 
with nuclear spins of nitrogen and hydrogen, as previously reported for the structurally identical thiophene compounds $\mathbf{I}^{+}$and $\mathbf{I I}^{+23}$ The hyperfine coupling constants are slightly higher for $\mathbf{1}^{+}$, $\mathbf{2}^{+}$, and $\mathbf{3}^{+}$than the values reported for their thiophene analogs $\mathbf{I}^{+}$and $\mathbf{~ I I}^{+}$(Table 5). The slight difference in hyperfine interaction might be due to the higher polarity of acetonitrile (used as solvent in the case of $\mathbf{1}^{+}, \mathbf{2}^{+}$, and $\mathbf{3}^{+}$) compared to dichloromethane (employed in our prior study of $\mathbf{I}^{+}$and $\mathbf{I I}^{+}$). The simulation of the EPR spectra for the monocation radicals $\mathbf{1}^{+}$and $\mathbf{3}^{+}$is similar to that reported for $\mathbf{I}^{+}$: the unpaired electron simultaneously interacts with two equivalent nitrogen nuclei (with $a_{\mathrm{N}}=4.7 \mathrm{G}$ for $\mathbf{1}^{+}$and $\mathbf{3}^{+}$), and two equivalent hydrogen nuclei (with $a_{\mathrm{H}}=$ $3.9 \mathrm{G}$ for $\mathbf{1}^{+}$, and $3.8 \mathrm{G}$ for $\mathbf{3}^{+}$, respectively). The $a_{\mathrm{N}}$ values of monocation radicals $\mathbf{1}^{+}$and $\mathbf{3}^{+}$are compatible with complete delocalization of the unpaired electron on the EPR timescale, i. e., with class III mixed-valence behavior. ${ }^{22}$ The simulation of the EPR spectrum for monocation radical $2^{+}$indicates a simultaneous interaction of the unpaired electron with two equivalent nitrogen nuclei with $a_{\mathrm{N}}=3.1 \mathrm{G}$, and two nonequivalent hydrogen nuclei with $a_{\mathrm{H} 1}=2.9 \mathrm{G}$ and $a_{\mathrm{H} 1}=2.1 \mathrm{G}$. Both the $a_{\mathrm{N}}$ value smaller than $4.5 \mathrm{G}$ and the magnetic nonequivalence of hydrogen nuclei interacting with the unpaired electron are compatible with class II mixed-valence behavior in radical $2^{+23}$

The nitrogen hyperfine constant decreases from $4.7 \mathrm{G}$ to $3.1 \mathrm{G}$ when going from $\mathbf{1}^{+}$to $\mathbf{2}^{+}$, and a similar observation has been made previously for $\mathbf{I}^{+}$and $\mathbf{I I}^{+23}$ This finding can be attributed to spin delocalization away from the nitrogen atoms towards the center of the chalcogenophene bridge. $^{22}$ It is likely that this phenomenon is caused by an increase in $\pi$-conjugation when going from the mono- to the bichalcogenophenes. In other words, with increasing bridge length the amino-decorated chalcogenophene monocations are to be considered more and more as bridgeoxidized rather than amino-oxidized species. The spin density on the selenophene bridge is 
increased at the expense of that on the nitrogen atom as also seen for alkoxyphenyl amino radical cations. $^{22}$ This interpretation is supported by density-functional theory (vide infra as well as Tables S6 and S7; note that the spin density is distributed symmetrically in the DFT results).

Table 5. EPR parameters ( $g$ factors and hyperfine coupling constants) for selenophene and thiophene cations.

\begin{tabular}{|c|c|c|c|c|}
\hline compd & $g$ & $a_{\mathrm{N}}[\mathrm{G}]$ & $a_{\mathrm{H} 1}[\mathrm{G}]$ & $a_{\mathrm{H} 2}[\mathrm{G}]$ \\
\hline $\mathbf{1}^{+a}$ & 2.0030 & 4.7 & 3.9 & \\
\hline $\mathbf{2}^{+a}$ & 2.0035 & 3.1 & 2.9 & 2.1 \\
\hline $\mathbf{3}^{+a}$ & 2.0030 & 4.7 & 3.8 & \\
\hline $\mathbf{I}^{+b}$ & 2.0050 & 4.3 & 2.6 & \\
\hline $\mathbf{I I}^{+b}$ & 2.0050 & 2.9 & 2.4 & 2.0 \\
\hline
\end{tabular}

${ }^{a}$ This work, measured in $\mathrm{CH}_{3} \mathrm{CN} ;{ }^{b}$ From ref. ${ }^{23}$, measured in $\mathrm{CH}_{2} \mathrm{Cl}_{2}$.

Quantum-chemical studies of oxidation potentials and intervalence charge transfer. On average, density-functional based calculations are the best compromise between computational cost and accuracy in quantum-chemical studies. Unfortunately, those methods tend to overdelocalize the system's electrons due to inherent self-interaction errors, which is particularly critical for mixed-valence compounds. ${ }^{11}$ For this purpose, Renz and Kaupp developed a protocol to avoid the problems without increasing the computational cost too much. ${ }^{32-34}$ The use of a hybrid functional with intermediate exact-exchange admixture (35-42\%) adjusts overdelocalization, whereas the use of the conductor-like polarizable continuum model (CPCM) is supposed to consider individual solvent polarities. It was shown that the protocol performs well for bis-triarylamine radical cations, neutral triarylamine-triarylmethyl radicals, dinitroaryl radical 
cations and diquinone radical anions. ${ }^{32-34}$ Our calculations show that in the course of oxidation to the mono- and the dicationic forms, our systems adopt an increasingly quinoidal structure, without any significant differences between thiophene and selenophene compounds (Tables S3 to S5).

Table 6 summarizes the relevant differences between measured and calculated vertical (VOP) and adiabatic (AOP) oxidation potentials (see Supporting Information for raw data and for more details on the definition of AOP and VOP), where we have assumed that the experimental values given in volts can be expressed as given in electron volts because one-electron processes are studied. Note that two different conformers of the bichalcogenophenes were employed in the calculations (Scheme S1). As their energies are almost the same in each case, we present, for the sake of brevity, Boltzmann-weighted averages with an assumed standard temperature throughout this work (the temperature is not considered in the calculations, but since the deviation to the arithmetic averages is less than $1 \%$, the use of Boltzmann weights is not an unreasonable choice). Energy values for the individual conformers are listed in the Supporting Information (individual energies obtained with the BMK hybrid functional are listed in Table S1).

Table 6. Differences between different kinds of experimentally $\left(\Delta \mathrm{E}_{\mathrm{ox}}\right)$ and computationally (BLYP35/TZVP/CPCM:MeCN) determined oxidation potentials. AOP and VOP are defined in the Supporting Information.

\begin{tabular}{|l|l|l|l|}
\hline $1^{\text {st }}$ oxidation potential & $\Delta \mathrm{AOP}[\mathrm{meV}]$ & $\Delta \mathrm{VOP}[\mathrm{meV}]$ & $\Delta \mathrm{E}_{\mathrm{ox}}[\mathrm{meV}]$ \\
\hline $\mathbf{2}-\mathbf{1}$ & 33.5 & -102 & $\sim 60$ \\
\hline $\mathbf{3}-\mathbf{1}$ & 409 & 502 & $\sim 320$ \\
\hline
\end{tabular}




\begin{tabular}{|l|l|l|l|}
\hline I $-\mathbf{I}$ & 33.4 & -70.0 & $\sim 60$ \\
\hline I - 1 & 44.7 & 51.6 & $\sim 0$ \\
\hline II - 2 & 44.7 & 83.8 & $\sim 0$ \\
\hline $2^{\text {nd }}$ oxidation potential & & & \\
\hline $\mathbf{2}-\mathbf{1}$ & -303 & -227 & $\sim-220$ \\
\hline $\mathbf{3}-\mathbf{1}$ & 335 & 372 & $\sim 250$ \\
\hline II - I & -275 & -210 & $\sim-140$ \\
\hline $\mathbf{I}-\mathbf{1}$ & 37.6 & 35.0 & $\sim 60$ \\
\hline II - 2 & 65.9 & 52.1 & $\sim 140$ \\
\hline $2^{\text {nd }}-1^{\text {st }}$ oxidation potential & & & \\
\hline $\mathbf{1}$ & 917 & 538 & 393 \\
\hline $\mathbf{2}$ & 581 & 412 & 109 \\
\hline $\mathbf{3}$ & 843 & 406 & 321 \\
\hline I & 910 & 521 & 450 \\
\hline II & 602 & 381 & 250 \\
\hline
\end{tabular}

Considering that the typical error for energy differences obtained from KS-DFT calculations is on the order of $5-10 \mathrm{~kJ} / \mathrm{mol}(\sim 50-100 \mathrm{meV})$, the relations of the first AOPs are remarkably close to the experimental benchmark, whereas those of the first VOPs are less close. The average relations of the second AOPs and VOPs are moderately close to the experimental results for the selenophene compounds and the monochalcogenophenes, and less close for the thiophene compounds and the bichalcogenophenes. Finally, the differences between the first and second oxidation potentials are very poorly reproduced by the computations compared to the 
experimental benchmark (even though the qualitative trends are roughly correct when using adiabatic oxidation potentials). Table S2 lists individual oxidation potentials obtained with BLYP35 and BMK hybrid functionals, respectively.

To interpret these results, it is important to keep in mind that the molecules in the experimental setup are exposed to an external electric field and to counter ions, which was not considered in the calculations. As the external field increases in the course of an oxidative potential sweep, the oxidation of the neutral compounds is presumably less influenced by this field than oxidation of the monocations (which requires higher potentials). Furthermore, spin analyses of the optimized monocations (Tables S6 and S7) reveal that the spin is significantly more distributed over the bridges of the bichalcogenophenes than over those of the monochalcogenophenes. This observation might explain why the oxidation-potential differences of the bichalcogenophenes are less close to the experiment than those of the monochalcogenophenes: Due to the relatively large polarizabilities of the chalcogen atoms, the external electric field is assumed to increasingly influence those compounds which reveal large spin densities at the bridge. However, this argumentation does not give a clue why the results of the selenophene compounds are closer to the experiment than those of the thiophene compounds. It might be that the oxidation potentials of the selenophene compounds change more linearly with the increase of the external field because the polarizability of selenophene is significantly lower than that of thiophene. ${ }^{66}$ This hypothesis is supported by the findings that (i) the computationally determined differences between first and second oxidation potentials (for a given species) differ significantly from those obtained by cyclic-voltammetry, but (ii) relations between different species for a given oxidation potential differ much less between computation and experiment, particularly for the selenophene compounds and monochalcogenophenes (Table 6). Introducing a single $\mathrm{PF}_{6}^{-}$anion and 
performing another structure optimization does not change the similarity of ionization potentials between thiophene and selenophene compounds (cf. Cartesian coordinates and Table S2 in the Supporting Information).

A different course to interpreting the results is to assume that the optimized structures do not represent the experimentally relevant ones. Therefore, we made different attempts to obtain the correct minimum on the potential energy surface. First, we employed two different types of asymmetric initial structures (see the Supporting Information) for the optimizations of the radical cations. Second, we additionally employed the BMK and BLYP80 hybrid functionals (both contain a larger exact-exchange admixture favoring electron localization). Third, we considered two different conformers for the bichalcogenophenes (Scheme S1), respectively. Fourth, we also performed optimizations for bichalcogenophenes with mutually orthogonal heterocycles to impede electronic communication. However, in all cases we obtained the same symmetric optimized structures revealing a completely delocalized unpaired electron, compatible with Robin-Day class III (see Tables S3-S5 for relevant bond lengths and dihedral angles of all molecular structures as a function of the total electric charge). Thus, there is much evidence that the optimized structures indeed represent the real equilibrium structures in acetonitrile (assuming that the combinations of exchange-correlation functionals and the solvation model employed are suitable for the problem under study, as suggested by refs. ${ }^{32-34}$ ).

To summarize, the calculated results (Table 6) confirm the experimental finding that the length of the bridge has a significantly stronger influence on the comproportionation constant than the electronic structure of the redox-active units. On the other hand, the large difference between the comproportionation constants of biselenophene and bithiophene is not reproduced by our KSDFT computations. It might be that this difference is only pronounced in the presence of an 
external electric field. The computed equivalence of the monocation stability does not necessarily mean that both biselenophene and bithiophene exhibit an equivalent extent of electronic communication, ${ }^{44,45}$ particularly because stability is a steady-state phenomenon whereas communication is a dynamic process. Transition dipole moments related to electronic excitations, which are not covered by time-independent computations, can play a decisive role in electronic communication. Quantum-chemical studies of the spectroscopic properties of the radical cations may provide an answer to this problem and serve to evaluate the quality of our previous structure optimizations.

For this purpose, we examined the IVCT bands of the radical cations from a computational perspective. To evaluate the calculations, we additionally computed the lowest-energy transition of the neutral compounds (Table S8) which do not exhibit intervalence charge transfers. The wavenumbers of the computed lowest-energy transitions match qualitatively with the experimental results, but are overestimated by about $2000 \mathrm{~cm}^{-1}$, which is an indication of a class III situation (Figure 6 and Table 7). ${ }^{38, a, c, d}$ Furthermore, we also observed a blue shift of the IVCT band from $\mathbf{1}^{+}$to $\mathbf{3}^{+}\left(16665 \mathrm{~cm}^{-1}\right.$ vs $\left.18259 \mathrm{~cm}^{-1}\right)$ employing BLYP35.
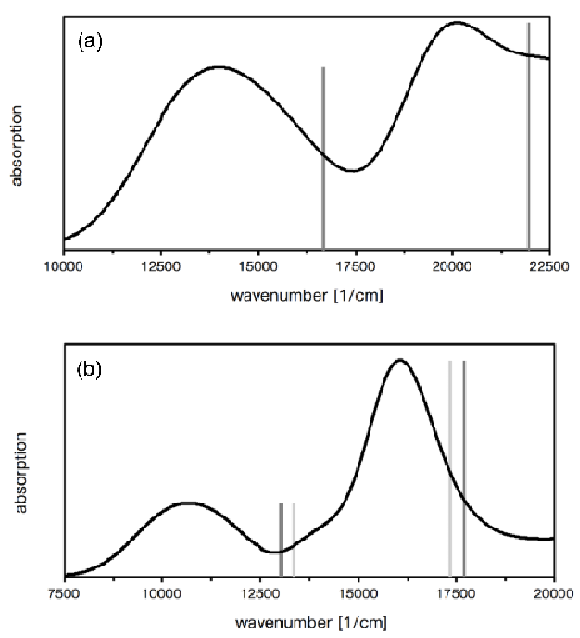
Figure 6. Experimental UV/Vis/NIR spectra (black lines) and computed (TD-DFT: BLYP35/TZVP/CPCM:MeCN) transitions (gray sticks) for (a) the monoselenophene radical cation $\mathbf{1}^{+}$and (b) the biselenophene radical cation $\mathbf{2}^{+}$(dark gray: SeCCSe dihedral angle of $180^{\circ}$; light gray: SeCCSe dihedral angle of $0^{\circ}$ ).

Table 7. Energy of ICVT band maxima (in wavenumbers) obtained from experiment and computations (TD-DFT: BLYP35;BMK;M06HF/TZVP/CPCM:MeCN). Optimized structures obtained from BLYP35 calculations were employed.

\begin{tabular}{|c|c|c|c|c|}
\hline cmpd & $\begin{array}{c}\text { ₹ै(max,exp }) \\
{\left[\mathrm{cm}^{-1}\right]}\end{array}$ & $\begin{array}{c}\text { พ}(\max , \mathrm{BLYP} 35) \\
{\left[\mathrm{cm}^{-1}\right]}\end{array}$ & $\begin{array}{c}\text { ₹}(\max , \mathrm{BMK}) \\
{\left[\mathrm{cm}^{-1}\right]}\end{array}$ & $\begin{array}{c}\text { ₹(max,M06HF }) \\
{\left[\mathrm{cm}^{-1}\right]}\end{array}$ \\
\hline $\mathbf{1}^{+}$ & 13970 & 16665 & 17252 & 21356 \\
\hline $\mathbf{2}^{+}$ & 10670 & 13180 & 13460 & 15624 \\
\hline $\mathbf{I}^{+}$ & 13850 & 16129 & 16659 & 20800 \\
\hline $\mathbf{I I}^{+}$ & 10620 & 12962 & 13171 & 15211 \\
\hline
\end{tabular}

In order to determine the extent of electronic communication in the studied compounds, it is important to investigate solvent effects and to explicitly calculate electronic coupling elements, particularly as the computations do not provide band widths and band asymmetries. In contrast to the experiment, the computational results for dichloromethane only reveal slight red shifts of about $200 \mathrm{~cm}^{-1}$ (Table S9) when compared to the results for acetonitrile. This may be due to the use of molecular structures optimized by employing the dielectric constant of acetonitrile also in the single-point calculations based on a dichloromethane solvent model. 
Table 8 summarizes the transition dipole moments of the lowest-energy transitions and N-N distances of optimized radical cations. The computed electronic coupling matrix elements reproduce the experimental trend (Figure 7), although the difference between biselenophene and bithiophene is significantly smaller compared to the experiment. This decrease also leads to a decay constant for the selenophenes $\left(0.33 \AA^{-1}\right)$ that is only slightly larger than that of the thiophenes $\left(0.30 \AA^{-1}\right)$. The M06HF hybrid functional suggests IVCT band maxima that are too high in energy compared to the results obtained from employing BLYP35 and BMK, respectively, which may be due to the use of molecular structures optimized with BLYP35.

Table 8. Computed transition dipole moments of the lowest-energy transition and N-N distances of optimized radical cations (TD-DFT:BLYP35;BMK;M06HF/TZVP/CPCM:MeCN). The values for the bichalcogenophene are averages due to the use of two different conformations.

\begin{tabular}{|l|l|l|l|l|}
\hline & $\begin{array}{l}\mu[\mathrm{D}] \\
(B L Y P 35)\end{array}$ & $\begin{array}{l}\mu[\mathrm{D}] \\
(B M K)\end{array}$ & $\begin{array}{l}\mu[\mathrm{D}] \\
(\mathrm{M} 06 \mathrm{HF})\end{array}$ & $\begin{array}{l}\mathrm{d}(\mathrm{N}-\mathrm{N})[\AA] \\
(\mathrm{BLYP35})\end{array}$ \\
\hline $\mathbf{1}^{+}$ & 7.3 & 7.2 & 6.9 & 5.23 \\
\hline $\mathbf{2}^{+}$ & 8.1 & 8.4 & 9.1 & 9.14 \\
\hline $\mathbf{I}^{+}$ & 7.6 & 7.5 & 7.3 & 5.11 \\
\hline $\mathbf{I I}^{+}$ & 9.3 & 9.4 & 9.9 & 8.84 \\
\hline
\end{tabular}

The striking result is that the selenophene radical cations reveal a smaller extent of electronic communication compared to the thiophene radical cations ( $4 \%$ for the mono compounds and $11 \%$ for the bi compounds on average, respectively), even though their stabilities (expressed in terms of comproportionation constants $\mathrm{K}_{\mathrm{c}}$ ) seem to be similar in absence of an external electric field (according to computation). In order to evaluate the quality of the optimized molecular 
structure of the biselenophene, whose computed electronic coupling element is still far away from the experimentally determined one, we modified the dihedral angle between the heterocycles of both conformers (Scheme S1) by only a few degrees, i.e., by $5^{\circ}, 10^{\circ}$ and $20^{\circ}$, respectively. The arithmetic average $H_{\mathrm{AB}}$ value (TD-DFT: BLYP35/TZVP/CPCM:MeCN) is found to be $2475(69) \mathrm{cm}^{-1}$ instead of $2434(40) \mathrm{cm}^{-1}$ (Figure 7) when taking only the two optimized conformers into account. This change is not significant, and we conclude that the computed structures for biselenophene are satisfactory within the frame of the method developed by Renz and Kaupp. It appears that more sophisticated solvent models, possibly combined with relativistic effects would have to be considered in order to reproduce the experimental trends quantitatively. A first calculation including spin-orbit interactions in combination with a pure exchange-correlation functional and neglecting solvent effects could not explain the observed experimental trends (Table S10), but further studies combining the Renz/Kaupp protocol with relativistic electronic-structure calculations may provide further insight. Renz and Kaupp also showed that the direct conductor-like screening model for real solvents (D-COSMO-RS) is a promising solvent model going beyond continuum models at small extra cost. ${ }^{33}$

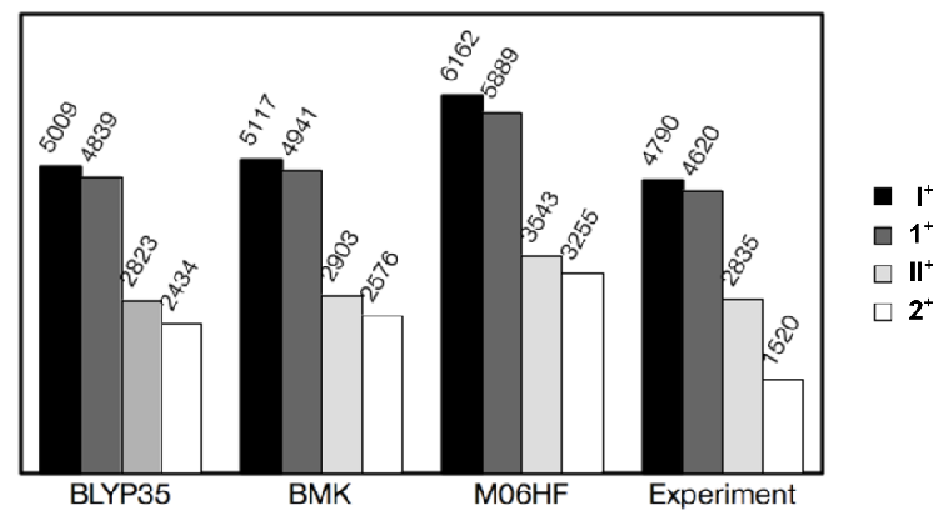

Figure 7. Experimentally and computationally BLYP35;BMK;M06HF/TZVP/CPCM:MeCN) determined electronic coupling matrix elements 
(in $\mathrm{cm}^{-1}$ ) for radical cations $\mathbf{1}^{+}, \mathbf{2}^{+}, \mathbf{I}^{+}$and $\mathbf{I I}^{+}$. Tables 7 and 8 list the explicit values used for the calculation of the presented couplings.

\section{SUMMARY AND CONCLUSIONS}

A combination of cyclic voltammetry, optical absorption, and EPR spectroscopy leads to the conclusion that the extent of charge delocalization in the monoselenophene and monothiophene cations $\mathbf{1}^{+}$and $\mathbf{I}^{+}$is similar. By contrast, in biselenophene $\mathbf{2}^{+}$the electronic coupling matrix element $H_{A B}$ is roughly $45 \%$ lower than in bithiophene $\mathbf{I I}^{+}$, and the comproportionation constant for compound $\mathbf{2}$ is a factor of 240 lower than that of compound II. These are rather dramatic differences given the structural similarity between these compounds. However, the computationally determined comproportionation constants of biselenophene and bithiophene are almost identical and the coupling element of the monocationic biselenophene is only about $15 \%$ smaller than that of the monocationic bithiophene. While it is reassuring that KS-DFT calculations thus suggest the same qualitative trend as the experiment, the underestimation of the decrease of communication in biselenophene $\mathbf{2}^{+}$along with deviations in UV/VIS absorption band positions may point to shortcomings in the employed exchange-correlation functionals and/or solvent models. It could also be due to a neglect of spin-orbit coupling in the calculations, and this could be problematic because spin-orbit coupling is considerably stronger for selenium than for sulfur. The deviating monocation stabilities suggested by cyclic-voltammetry measurements and KS-DFT calculations may be caused by the external electric field employed in the experiment (which is not considered in the calculations). 
It is not possible to extrapolate from our mono- and biselenophenes to polyselenophene. However, to put things carefully, from our study it would appear that the exchange of the chalcogenophene heteroatom from sulfur to selenium is not necessarily associated with a benefit in terms of charge transfer, at least in short oligomeric structures.

\section{ASSOCIATED CONTENT}

Synthetic protocols and characterization data for molecules $\mathbf{1}-\mathbf{4}$ and all intermediate reaction products. Additional cyclic voltammetry data. Derivation of the equations for the distance decay constants $\beta$ and $\beta_{\mathrm{n}}$. Additional details on quantum-chemical software and computational settings. Calculated molecular Cartesian coordinates, local spins and UV/Vis data for the neutral compounds. This material is available free of charge via the Internet at http://pubs.acs.org.

\section{AUTHOR INFORMATION}

Corresponding Author

*E-mail: carmen.herrmann@chemie.uni-hamburg.de, oliver.wenger@unibas.ch

\section{ACKNOWLEDGMENT}

This work was supported by the Swiss NSF through grant number 200021_146231/1.

J.P. and C.H. acknowledge computational resources of the high-performance computing center (RRZ) of the University of Hamburg. 


\section{REFERENCES}

(1) Gunes, S.; Neugebauer, H.; Sariciftci, N. S. Conjugated Polymer-Based Organic Solar Cells. Chem. Rev. 2007, 107, 1324-1338.

(2) Grimsdale, A. C.; Chan, K. L.; Martin, R. E.; Jokisz, P. G.; Holmes, A. B. Synthesis of Light-Emitting Conjugated Polymers for Applications in Electroluminescent Devices. Chem. Rev. 2009, 109, 897-1091.

(3) Roncali, J. Conjugaed (Poly)thiophenes - Synthesis, Functionalization, and Applications. Chem. Rev. 1992, 92, 711-738.

(4) Gidron, O.; Dadvand, A.; Sun, E. W. H.; Chung, I.; Shimon, L. J. W.; Bendikov, M.; Perepichka, D. F. Oligofuran-Containing Molecules for Organic Electronics. J. Mater. Chem. C 2013, 1, 4358-4367.

(5) Gidron, O.; Diskin-Posner, Y.; Bendikov, M. High Charge Delocalization and Conjugation in Oligofuran Molecular Wires. Chem.-Eur. J. 2013, 19, 13140-13150.

(6) Patra, A.; Bendikov, M. Polyselenophenes. J. Mater. Chem. 2010, 20, 422-433.

(7) Patra, A.; Wijsboom, Y. H.; Zade, S. S.; Li, M.; Sheynin, Y.; Leitus, G.; Bendikov, M. Poly(3,4-ethylenedioxyselenophene). J. Am. Chem. Soc. 2008, 130, 6734-6735.

(8) Li, L. S.; Hollinger, J.; Jahnke, A. A.; Petrov, S.; Seferos, D. S. Polyselenophenes with Distinct Crystallization Properties. Chem. Sci. 2011, 2, 2306-2310.

(9) Jahnke, A. A.; Howe, G. W.; Seferos, D. S. Polytellurophenes with Properties Controlled by Tellurium-Coordination. Angew. Chem. Int. Ed. 2010, 49, 10140-10144. 
(10) Jahnke, A. A.; Seferos, D. S. Polytellurophenes. Macromol. Rapid Commun. 2011, 32, 943-951.

(11) Haid, S.; Mishra, A.; Uhrich, C.; Pfeiffer, M.; Bäuerle, P. DicyanovinyleneSubstituted Selenophene-Thiophene Co-oligomers for Small-Molecule Organic Solar Cells. Chem. Mater. 2011, 23, 4435-4444.

(12) Heckmann, A.; Lambert, C. Organic Mixed-Valence Compounds: A Playground for Electrons and Holes. Angew. Chem. Int. Ed. 2012, 51, 326-392.

(13) Hankache, J.; Wenger, O. S. Organic Mixed Valence. Chem. Rev. 2011, 111, 5138-5178.

(14) Lacroix, J. C.; Chane-Ching, K. I.; Maquère, F.; Maurel, F. Intrachain Electron Transfer in Conducting Oligomers and Polymers: The Mixed Valence Approach. J. Am. Chem. Soc. 2006, 128, 7264-7276.

(15) Lloveras, V.; Vidal-Gancedo, J.; Figueira-Duarte, T. M.; Nierengarten, J. F.; Novoa, J. J.; Mota, F.; Ventosa, N.; Rovira, C.; Veciana, J. Tunneling versus Hopping in MixedValence Oligo-p-phenylenevinylene Polychlorinated Bis(triphenylmethyl) Radical Anions. J. Am. Chem. Soc. 2011, 133, 5818-5833.

(16) Zhu, Y. B.; Wolf, M. O. Charge Transfer and Delocalization in Conjugated (Ferrocenylethynyl)oligothiophene Complexes. J. Am. Chem. Soc. 2000, 122, 10121-10125.

(17) Rohde, D.; Dunsch, L.; Tabet, A.; Hartmann, H.; Fabian, J. Radical Ions of $\alpha, \alpha^{\prime}-$ Bis(Diphenylamino)-Capped Oligothiophenes: A Combined Spectroelectrochemical and Theoretical Study. J. Phys. Chem. B 2006, 110, 8223-8231. 
(18) Bäuerle, P.; Segelbacher, U.; Maier, A.; Mehring, M. Electronic Structure of Monomeric and Dimeric Cation Radicals in End-Capped Oligothiophenes. J. Am. Chem. Soc. 1993, 115, 10217-10223.

(19) Casado, J.; Gonzalez, S. R.; Delgado, M. C. R.; Oliva, M. M.; Navarrete, J. T. L.; Caballero, R.; de la Cruz, P.; Langa, F. Ferrocenyl-Ended Thieno-Vinylene Oligomers: DonorAcceptor Polarization and Mixed-Valence Properties with Emphasis on the Raman Mapping of Localized-to-Delocalized Transitions. Chem.-Eur. J. 2009, 15, 2548-2559.

(20) Le Stang, S.; Paul, F.; Lapinte, C. Molecular wires: Synthesis and Properties of the New Mixed-Valence Complex Cp*(dppe)Fe-C C-X-C C-Fe(dppe)Cp* $\mathrm{PF}_{6}\left(\mathrm{X}=2,5-\mathrm{C}_{4} \mathrm{H}_{2} \mathrm{~S}\right)$ and Comparison of its Properties with Those of the Related All-Carbon-Bridged Complex (X = $\left.\mathrm{C}_{4}-\right)$. Organometallics 2000, 19, 1035-1043.

(21) Nöll, G.; Avola, M.; Lynch, M.; Daub, J. Comparison of Alternant and Nonalternant Aromatic Bridge Systems with Respect to Their ET-Properties. J. Phys. Chem. C 2007, 111, 3197-3204.

(22) Odom, S. A.; Lancaster, K.; Beverina, L.; Lefler, K. M.; Thompson, N. J.; Coropceanu, V.; Brédas, J. L.; Marder, S. R.; Barlow, S. Bis Bis-(4-alkoxyphenyl) Amino Derivatives of Dithienylethene, Bithiophene, Dithienothiophene and Dithienopyrrole: PalladiumCatalysed Synthesis and Highly Delocalised Radical Cations. Chem. Eur. J. 2007, 13, 96379646.

(23) Reuter, L. G.; Bonn, A. G.; Stückl, A. C.; He, B. C.; Pati, P. B.; Zade, S. S.; Wenger, O. S. Charge Delocalization in a Homologous Series of $\alpha, \alpha^{\prime}$-Bis(dianisylamino)Substituted Thiophene Monocations. J. Phys. Chem. A 2012, 116, 7345-7352. 
(24) Zhang, F.; Gotz, G.; Mena-Osteritz, E.; Weil, M.; Sarkar, B.; Kaim, W.; Bäuerle, P. Molecular and Electronic Structure of Cyclo 10 Thiophene in Various Oxidation States: Polaron Pair vs. Bipolaron. Chem. Sci. 2011, 2, 781-784.

(25) Jenart, M.; Niebel, C.; Balandier, J. Y.; Leroy, J.; Mignolet, A.; Stas, S.; Van Vooren, A.; Cornil, J.; Geerts, Y. H. Quaterthiophene-Based Dimers Containing an Ethylene Bridge: Molecular Design, Synthesis, and Optoelectronic Properties. Tetrahedron 2012, 68, 349355.

(26) Zade, S. S.; Bendikov, M. Study of Hopping Transport in Long Oligothiophenes and Oligoselenophenes: Dependence of Reorganization Energy on Chain Length. Chem.-Eur. J. 2008, 14, 6734-6741.

(27) Jahnke, A. A.; Spulber, M.; Neuburger, M.; Palivan, C. G.; Wenger, O. S. Electronic Coupling Mediated by Furan, Thiophene, Selenophene and Tellurophene in a Homologous Series of Organic Mixed Valence Compounds. Chem. Commun. 2014, 50, 1088310886.

(28) Hildebrandt, A.; Lang, H. (Multi)ferrocenyl Five-Membered Heterocycles: Excellent Connecting Units for Electron Transfer Studies. Organometallics 2013, 32, 5640-5653.

(29) D'Alessandro, D. M.; Keene, F. R. Intervalence Charge Transfer (IVCT) in Trinuclear and Tetranuclear Complexes of Iron, Ruthenium, and Osmium. Chem. Rev. 2006, $106,2270-2298$.

(30) Fraysse, S.; Coudret, C.; Launay, J.-P. Molecular Wires Built from Binuclear Cyclometalated Complexes. J. Am. Chem. Soc. 2003, 125, 5880-5888. 
(31) Low, P. J.; Paterson, M. A. J.; Puschmann, H.; Goeta, A. E.; Howard, J. A. K.; Lambert, C.; Cherryman, J. C.; Tackley, D. R.; Leeming, S.; Brown, B. Crystal, Molecular and Electronic Structure of N,N'-diphenyl-N,N'-Bis(2,4-Dimethylphenyl)-(1,1'-Biphenyl)-4,4'Diamine and the Corresponding Radical Cation. Chem. Eur. J. 2004, 10, 83-91.

(32) Renz, M.; Theilacker, K.; Lambert, C.; Kaupp, M. A Reliable Quantum-Chemical Protocol for the Characterization of Organic Mixed-Valence Compounds. J. Am. Chem. Soc. 2009, 131, 16292-16302.

(33) Renz, M.; Kess, M.; Diedenhofen, M.; Klamt, A.; Kaupp, M. Reliable Quantum Chemical Prediction of the Localized/Delocalized Character of Organic Mixed-Valence Radical Anions. From Continuum Solvent Models to Direct-COSMO-RS. J. Chem. Theory Comput. 2012, 8, 4189-4203.

(34) Renz, M.; Kaupp, M. Predicting the Localized/Delocalized Character of MixedValence Diquinone Radical Anions. Toward the Right Answer for the Right Reason. J. Phys. Chem. A 2012, 116, 10629-10637.

(35) Becke, A. D. Density-Functional Exchange-Energy Approximation with Correct Asymptotic Behavior. Phys. Rev. A 1988, 38, 3098-3100.

(36) Lee, C. T.; Yang, W. T.; Parr, R. G. Development of the Colle-Salvetti Correlation-Energy Formula into a Functional of the Electron-Density. Phys. Rev. B 1988, 37, 785-789. 
(37) Miehlich, B.; Savin, A.; Stoll, H.; Preuss, H. Results Obtained with the Correlation-Energy Density Functionals of Becke and Lee, Yang and Parr. Chem. Phys. Lett. 1989, 157, 200-206.

(38) Schäfer, A.; Huber, C.; Ahlrichs, R. Fully Optimized Contracted Gaussian-Basis Sets of Triple Zeta Valence Quality for Atoms Li to Kr. J. Chem. Phys. 1994, 100, 5829-5835.

(39) Barone, V.; Cossi, M. Quantum Calculation of Molecular Energies and Energy Gradients in Solution by a Conductor Solvent Model. J. Phys. Chem. A 1998, 102, 1995-2001.

(40) Cossi, M.; Rega, N.; Scalmani, G.; Barone, V. Energies, Structures, and Electronic Properties of Molecules in Solution with the C-PCM Solvation Model. J. Comput. Chem. 2003, 24, 669-681.

(41) Boese, A. D.; Martin, J. M. L. Development of Density Functionals for Thermochemical Kinetics. J. Chem. Phys. 2004, 121, 3405-3416.

(42) Zhao, Y.; Truhlar, D. G. Comparative DFT Study of van der Waals Complexes: Rare-Gas Dimers, Alkaline-Earth Dimers, Zinc Dimer, and Zinc-Rare-Gas Dimers. J. Phys. Chem. A 2006, 110, 5121-5129.

(43) Zhao, Y.; Truhlar, D. G. Density Functional for Spectroscopy: No Long-Range Self-Interaction Error, Good Performance for Rydberg and Charge-Transfer States, and Better Performance on Average than B3LYP for Ground States. J. Phys. Chem. A 2006, 110, 1312613130.

(44) Kaim, W.; Klein, A.; Glöckle, M. Exploration of Mixed Valence Chemistry: Inventing New Analogues of the Creutz-Taube Ion. Acc. Chem. Res. 2000, 33, 755-763. 
(45) D'Alessandro, D. M.; Keene, F. R. A Cautionary Warning on the Use of Electrochemical Measurements to Calculate Comproportionation Constants for Mixed-Valence Compounds. Dalton Trans. 2004, 3950-3954.

(46) Lambert, C.; Nöll, G. The Class II/III Transition in Triarylamine Redox Systems. J. Am. Chem. Soc. 1999, 121, 8434-8442.

(47) Measurements made in presence of a shortfall of oxidant lead to identical extinction coefficents.

(48) Robin, M. B.; Day, P. Mixed Valence Chemistry. Adv. Inorg. Chem. Radiochem. $1967,10,247-422$.

(49) Hush, N. S. Intervalence-Transfer Absorption. Part 2. Theoretical Considerations and Spectroscopic Data. Prog. Inorg. Chem. 1967, 8, 391-444.

(50) D'Alessandro, D. M.; Keene, F. R. Current Trends and Future Challenges in the Experimental, Theoretical and Computational Analysis of Intervalence Charge Transfer (IVCT) Transitions. Chem. Soc. Rev. 2006, 35, 424-440.

(51) Brunschwig, B. S.; Creutz, C.; Sutin, N. Optical Transitions of Symmetrical Mixed-Valence Systems in the Class II-III Transition Regime. Chem. Soc. Rev. 2002, 31, 168184.

(52) Demadis, K. D.; Hartshorn, C. M.; Meyer, T. J. The Localized-to-Delocalized Transition in Mixed-Valence Chemistry. Chem. Rev. 2001, 101, 2655-2685.

(53) Lancaster, K.; Odom, S. A.; Jones, S. C.; Thayumanavan, S.; Marder, S. R.; Brédas, J. L.; Coropceanu, V.; Barlow, S. Intramolecular Electron-Transfer Rates in Mixed- 
Valence Triarylamines: Measurement by Variable-Temperature ESR Spectroscopy and Comparison with Optical Data. J. Am. Chem. Soc. 2009, 131, 1717-1723.

(54) Nelsen, S. F.; Konradsson, A. E.; Weaver, M. N.; Telo, J. P. Intervalence Near-IR Spectra of Delocalized Dinitroaromatic Radical Anions. J. Am. Chem. Soc. 2003, 125, 1249312501.

(55) Nelsen, S. F.; Weaver, M. N.; Zink, J. I.; Telo, J. P. Optical Spectra of Delocalized Dinitroaromatic Radical Anions Revisited. J. Am. Chem. Soc. 2005, 127, 1061110622.

(56) Kaupp, M.; Renz, M.; Parthey, M.; Stolte, M.; Würthner, F.; Lambert, C. Computational and Spectroscopic Studies of Organic Mixed-Valence Compounds: Where is the Charge? Phys. Chem. Chem. Phys. 2011, 13, 16973-16986.

(57) Nelsen, S. F.; Newton, M. D. Estimation of Electron Transfer Distances from AM1 Calculations. J. Phys. Chem. A 2000, 104, 10023-10031.

(58) McConnell, H. M. Intramolecular Charge Transfer In Aromatic Free Radicals. $J$. Chem. Phys. 1961, 35, 508-515.

(59) Gray, H. B.; Winkler, J. R. Long-Range Electron Transfer. Proc. Natl. Acad. Sci. U. S. A. 2005, 102, 3534-3539.

(60) Wenger, O. S. Photoinduced Electron and Energy Transfer in Phenylene Oligomers. Chem. Soc. Rev. 2011, 40, 3538-3550. 
(61) Weiss, E. A.; Ahrens, M. J.; Sinks, L. E.; Gusev, A. V.; Ratner, M. A.; Wasielewski, M. R. Making a Molecular Wire: Charge and Spin Transport through ParaPhenylene Oligomers. J. Am. Chem. Soc. 2004, 126, 5577-5584.

(62) Hanss, D.; Walther, M. E.; Wenger, O. S. Importance of Covalence, Conformational Effects and Tunneling-Barrier Heights for Long-Range Electron Transfer: Insights from Dyads with Oligo- $p$-phenylene, Oligo- $p$-xylene and Oligo- $p$-dimethoxybenzene Bridges. Coord. Chem. Rev. 2010, 254, 2584-2592.

(63) Albinsson, B.; Eng, M. P.; Pettersson, K.; Winters, M. U. Electron and Energy Transfer in Donor-Acceptor Systems with Conjugated Molecular Bridges. Phys. Chem. Chem. Phys. 2007, 9, 5847-5864.

(64) Wenger, O. S. How Donor-Bridge-Acceptor Energetics Influence Electron Tunneling Dynamics and Their Distance Dependences. Acc. Chem. Res. 2011, 44, 25-35.

(65) Lambert, C.; Nöll, G.; Schelter, J. Bridge-Mediated Hopping or Superexchange Electron-Transfer Processes in Bis(triarylamine) Systems. Nat. Mater. 2002, 1, 69-73.

(66) Millefiori, S.; Alparone, A. Theoretical Determination of the Vibrational and Electronic (Hyper)polarizabilities of $\mathrm{C}_{4} \mathrm{H}_{4} \mathrm{X}(\mathrm{X}=\mathrm{O}, \mathrm{S}, \mathrm{Se}, \mathrm{Te})$ Heterocycles. Phys. Chem. Chem. Phys. 2000, 11, 2495-2501. 


\section{TOC SYNOPSIS}

Charge delocalization in amino-decorated thiophene and selenophene monocations is similar but there are significant differences between analogous bithiophene and biselenophene monocations.

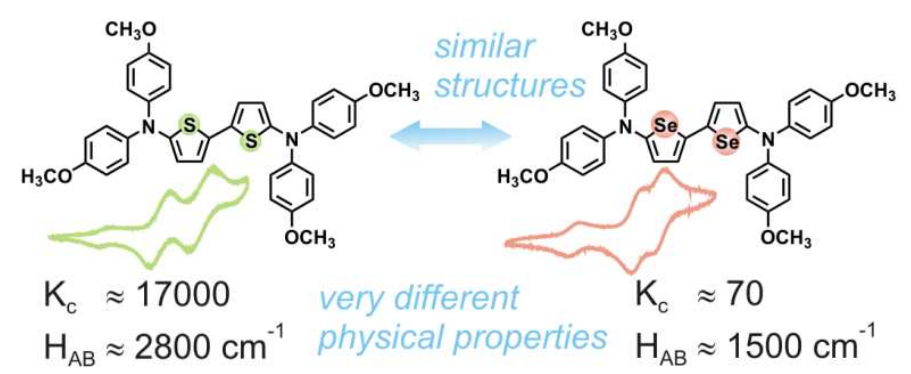

